\title{
A NOTE ON SOME GENERALIZED CLOSURE AND INTERIOR OPERATORS IN A TOPOLOGICAL SPACE
}

\author{
ANKIT GUPTA AND RATNA DEV SARMA
}

\begin{abstract}
If $X$ is a topological space and $A \subseteq X$, then the number of distinct sets that can be obtained from $A$ by using all possible compositions for operators $i_{\gamma}, c_{\gamma}$ (where $\gamma=\sigma, \pi, \alpha, \beta$ ) introduced by Császár is at the most 25. Explicit expressions for these sets are provided. An example is provided where all the 25 different sets are determined. The result is also discussed for special cases such as when the space is extremally disconnected, resolvable, open-unresolvable, and partition spaces.
\end{abstract}

\section{INTRODUCTION}

Kuratowski's closure complement theorem (also known as 14 set theorem) [11] has been a guiding source of research not only in topology, but also in various other fields such as Approximation Theory, Relational algebra, Formal Language, Computer programming $[4,5,9,15]$, etc. Peleg [15], while investigating the transitive closure of a binary relation, came across several closure operators which do not satisfy some of the four of Kuratowski's closure axioms, though their properties suffice to maintain "closure complement phenomenon". Similar kind of generalized closure operators are generated by the monotonic mappings introduced by Á. Császár [6]. When several such operators are considered simultaneously and composed, the study of closure complement phenomenon becomes complicated and highly interesting. In the present note, we provide our investigations regarding all possible compositions of four such generalized closure operators and their corresponding interior operators.

Let $X$ be a non empty set and let $\gamma: \mathcal{P}(X) \rightarrow \mathcal{P}(X)$ satisfy $\gamma(A) \subseteq \gamma(B)$ for $A \subseteq B$, where $\mathcal{P}(X)$ is the power set of $X$. According to Á. Császár [6], $A \subseteq X$ is called $\gamma$-open if $A \subseteq \gamma(A)$. The $\gamma$-open sets of $X$ form a generalized topology [8] (GT in brief) on $X$ in the sense that (i) $\phi$ is $\gamma$-open and (ii) any union of $\gamma$-open sets is again $\gamma$-open. A set is called $\gamma$-closed if its complement is $\gamma$-open. For $A \subseteq X$, the largest $\gamma$-open set contained in $A$ is called the $\gamma$-interior of $A$ and is denoted by $i_{\gamma}(A)$. Similarly, the smallest $\gamma$-closed set containing $A$ is called the $\gamma$-closure of $A$ and is denoted by $c_{\gamma}(A)$.

If $X$ is a topological space and $i$ and $c$ denote the interior and closure operators respectively, then $\gamma=c i, i c i, i c$ and $c i c$ give rise to important families of $\gamma$-open sets. The corresponding $\gamma$-open sets are known as semi-open, $\alpha$-open, $\pi$-open

$M S C$ (2010): primary 54A05; secondary 54A99.

Keywords: generalized open sets, $\sigma$-open, $\pi$-open, $\alpha$-open, $\beta$-open sets, closure operators, interior operators. 
and $\beta$-open sets respectively, in the literature. In [7], Á. Császár has shown that in some cases (including the four classical cases mentioned above), the $\gamma$-interior $i_{\gamma}(A)$ and the $\gamma$-closure $c_{\gamma}(A)$ of $A$ are easily obtained by explicit formulas. In the present note, we provide explicit formulas arising out of all possible compositions of different $i_{\gamma}$ 's and $c_{\gamma}$ 's, where $\gamma=c i, i c i, i c$, or $c i c$ in the topological framework. We have found that for $A \subseteq X$, from the possible compositions of $i_{\alpha}, i_{\sigma}, i_{\pi}, i_{\beta}$, $c_{\alpha}, c_{\sigma}, c_{\pi}$ and $c_{\beta}$, we get at most 25 different sets. We have provided explicit expressions of all these sets. We have also provided an example where this bound is achieved. If the topology satisfies some extra properties such as in an extremally disconnected space, $O U$-space and Partition space etc. the upper bound is less than 25. We have provided a discussion on such particular cases in the paper. Unlike in Kuratowski's 14 set theorem, these 25 operators do not form a monoid, but, rather, a semi-group. Algebraic properties of each of $i_{\gamma}, c_{\gamma}$ are investigated in our paper [17].

\section{THE MAIN RESULT}

First of all, we provide some definitions and notations:

Definition 2.1. Let $(X, \tau)$ be a topological space and $A \subseteq X$. Then, $A$ is called

(i) semi-open [12] if $A \subseteq \operatorname{cl}(\operatorname{int}(A))$;

(ii) $\alpha$-open [14] if $A \subseteq \operatorname{int}(\operatorname{cl}(\operatorname{int}(A)))$;

(iii) pre-open [13] if $A \subseteq \operatorname{int}(\operatorname{cl}(A))$;

(iv) $\beta$-open [1] if $A \subseteq \operatorname{cl}(\operatorname{int}(\operatorname{cl}(A)))$.

The complement of a semi-open set is called semi-closed. The largest semi-open set contained in $A$ is denoted by $i_{\sigma}(A)$. The smallest semi-closed set containing $A$ is denoted by $c_{\sigma}(A)$. Thus, the concept of a semi-open set gives rise to the operators $c_{\sigma}$ and $i_{\sigma}$. In an analogous way, the concept of an $\alpha$-open set gives rise to the operators $c_{\alpha}$ and $i_{\alpha}$, the concept of a pre-open set gives rise to the operators $c_{\pi}$ and $i_{\pi}$, and the concept of a $\beta$-open set gives rise to the operators $c_{\beta}$ and $i_{\beta}$.

Theorem 2.2. [7] In a topological space $(X, \tau)$ with $A \subseteq X$, we have,

(i) $c_{\sigma}(A)=A \cup \operatorname{int}(\operatorname{cl}(A))$;

(ii) $i_{\sigma}(A)=A \cap \operatorname{cl}(\operatorname{int}(A))$;

(iii) $c_{\alpha}(A)=A \cup \operatorname{cl}(\operatorname{int}(\operatorname{cl}(A)))$;

(iv) $i_{\alpha}(A)=A \cap \operatorname{int}(\operatorname{cl}(\operatorname{int}(A)))$;

(v) $c_{\pi}(A)=A \cup \operatorname{cl}(\operatorname{int}(A))$;

(vi) $i_{\pi}(A)=A \cap \operatorname{int}(\operatorname{cl}(A))$;

(vii) $c_{\beta}(A)=A \cup \operatorname{int}(\operatorname{cl}(\operatorname{int}(A)))$;

(viii) $i_{\beta}(A)=A \cap \operatorname{cl}(\operatorname{int}(\operatorname{cl}(A)))$.

The following result will be used to prove the main theorem:

Lemma 2.3. In a topological space $(X, \tau)$ with $A \subseteq X$, the following hold:

$$
\begin{aligned}
& \operatorname{cl}[A \cap \operatorname{int}(\operatorname{cl}(\operatorname{int}(A)))]=\operatorname{cl}(\operatorname{int}(A)) ; \\
& \operatorname{int}[A \cup \operatorname{cl}(\operatorname{int}(\operatorname{cl}(A)))]=\operatorname{int}(\operatorname{cl}(A)) ;
\end{aligned}
$$




$$
\begin{aligned}
\operatorname{cl}[A \cap \operatorname{cl}(\operatorname{int}(A))] & =\operatorname{cl}(\operatorname{int}(A)) ; \\
\operatorname{int}[A \cup \operatorname{int}(\operatorname{cl}(A))] & =\operatorname{int}(\operatorname{cl}(A)) ; \\
\operatorname{cl}[A \cap \operatorname{int}(\operatorname{cl}(A))] & =\operatorname{cl}(\operatorname{int}(\operatorname{cl}(A))) ; \\
\operatorname{int}[A \cup \operatorname{cl}(\operatorname{int}(A))] & =\operatorname{int}(\operatorname{cl}(\operatorname{int}(A))) ; \\
\operatorname{cl}[A \cap \operatorname{cl}(\operatorname{int}(\operatorname{cl}(A)))] & =\operatorname{cl}(\operatorname{int}(\operatorname{cl}(A))) ; \\
\operatorname{int}[A \cup \operatorname{int}(\operatorname{cl}(\operatorname{int}(A)))] & =\operatorname{int}(\operatorname{cl}(\operatorname{int}(A))) ; \\
\operatorname{cl}[A \cap \operatorname{cl}(\operatorname{int}(A)) \cap \operatorname{int}(\operatorname{cl}(A))] & =\operatorname{cl}(\operatorname{int}(A)) ; \\
\operatorname{int}[A \cup \operatorname{int}(\operatorname{cl}(A)) \cup \operatorname{cl}(\operatorname{int}(A))] & =\operatorname{int}(\operatorname{cl}(A)) ; \\
\operatorname{cl}[\operatorname{cl}(\operatorname{int}(A)) \cap \operatorname{int}(\operatorname{cl}(A))] & =\operatorname{cl}(\operatorname{int}(A)) ; \\
\operatorname{int}[\operatorname{cl}(\operatorname{int}(A)) \cup \operatorname{int}(\operatorname{cl}(A))] & =\operatorname{int}(\operatorname{cl}(A)) .
\end{aligned}
$$

Proof. Let $(X, \tau)$ be a topological space and $A \subseteq X$. Then, $\operatorname{cl}[A \cap \operatorname{int}(\operatorname{cl}(\operatorname{int}(A)))]$ $\subseteq \operatorname{cl}(A) \cap \operatorname{cl}(\operatorname{int}(\operatorname{cl}(\operatorname{int}(A)))) \subseteq \operatorname{cl}(A) \cap \operatorname{cl}(\operatorname{int}(A))=\operatorname{cl}(\operatorname{int}(A))$. Again, consider $\operatorname{int}(A) \subseteq A$ and $\operatorname{int}(A) \subseteq \operatorname{int}(\operatorname{cl}(\operatorname{int}(A)))$, thus $\operatorname{int}(A) \subseteq A \cap \operatorname{int}(\operatorname{cl}(\operatorname{int}(A)))$. Hence, $\operatorname{cl}(\operatorname{int}(A)) \subseteq \operatorname{cl}[A \cap \operatorname{int}(\operatorname{cl}(\operatorname{int}(A)))]$. Therefore, $\operatorname{cl}[A \cap \operatorname{int}(\operatorname{cl}(\operatorname{int}(A)))]=\operatorname{cl}(\operatorname{int}(A))$. One can prove all the other equalities in an analogous way.

Now we come to the main result:

Theorem 2.4. Let $(X, \tau)$ be a topological space and $A \subseteq X$. Then, the total number of distinct sets that can be obtained from $A$ by repeatedly using the operators $i_{\gamma}, c_{\gamma}$ (where $\left.\gamma=\sigma, \pi, \alpha, \beta\right)$ is at most 25 and there exists a topological space $(X, \tau)$ and a set $A \subseteq X$ from which all these 25 sets can be realized.

Proof. In a topological space $X$ with $A \subseteq X$, we have

$$
\begin{aligned}
i_{\sigma} \circ i_{\sigma}(A)=i_{\sigma}\left(i_{\sigma}(A)\right) & =i_{\sigma}(A \cap \operatorname{cl}(\operatorname{int}(A))) \\
& =(A \cap \operatorname{cl}(\operatorname{int}(A))) \cap \operatorname{cl}[\operatorname{int}(A \cap \operatorname{cl}(\operatorname{int}(A)))] \\
& =(A \cap \operatorname{cl}(\operatorname{int}(A))) \cap \operatorname{cl}[\operatorname{int}(A) \cap \operatorname{int}(\operatorname{cl}(\operatorname{int}(A)))] \\
& =(A \cap \operatorname{cl}(\operatorname{int}(A))) \cap \operatorname{cl}[\operatorname{int}(A)] \\
& =A \cap \operatorname{cl}(\operatorname{int}(A)) \\
& =i_{\sigma}(A) .
\end{aligned}
$$

We express this by $i_{\sigma} \circ i_{\sigma}=i_{\sigma}$. In the case of $i_{\sigma}$ and $i_{\pi}$, we have

$$
\begin{aligned}
i_{\sigma} \circ i_{\pi}(A) & =i_{\sigma}(A \cap \operatorname{int}(\operatorname{cl}(A))) \\
& =(A \cap \operatorname{int}(\operatorname{cl}(A))) \cap \operatorname{cl}[\operatorname{int}(A \cap \operatorname{int}(\operatorname{cl}(A)))] \\
& =(A \cap \operatorname{int}(\operatorname{cl}(A))) \cap \operatorname{cl}[\operatorname{int}(A) \cap \operatorname{int}(\operatorname{int}(\operatorname{cl}(A)))] \\
& =A \cap \operatorname{int}(\operatorname{cl}(A)) \cap \operatorname{cl}(\operatorname{int}(A)) .
\end{aligned}
$$

We express this by $i_{\sigma} \circ i_{\pi}=A \cap \operatorname{int}(\operatorname{cl}(A)) \cap \operatorname{cl}(\operatorname{int}(A))$.

We put together similar results by using the following composition tables. For convenience, we write $\mathrm{c}$ and $\mathrm{i}$ for $\mathrm{cl}$ and int respectively.

From Tables $1-4$, it is clear that so far 21 distinct sets have been realized from a given set $A$ by the compositions listed in the tables. We enumerate them as 
Table 1

\begin{tabular}{|c||c|c|c|c|}
\hline$\circ$ & $i_{\sigma}$ & $i_{\alpha}$ & $i_{\pi}$ & $i_{\beta}$ \\
\hline \hline$i_{\sigma}$ & $i_{\sigma}$ & $i_{\alpha}$ & $A \cap \operatorname{ic}(A) \cap \operatorname{ci}(A)$ & $i_{\sigma}$ \\
\hline$i_{\alpha}$ & $i_{\alpha}$ & $i_{\alpha}$ & $i_{\alpha}$ & $i_{\alpha}$ \\
\hline$i_{\pi}$ & $i_{\alpha}$ & $i_{\alpha}$ & $i_{\pi}$ & $i_{\pi}$ \\
\hline$i_{\beta}$ & $i_{\sigma}$ & $i_{\alpha}$ & $i_{\pi}$ & $i_{\beta}$ \\
\hline
\end{tabular}

Table 2

\begin{tabular}{|c||c|c|c|c|}
\hline$\circ$ & $c_{\sigma}$ & $c_{\alpha}$ & $c_{\pi}$ & $c_{\beta}$ \\
\hline \hline$c_{\sigma}$ & $c_{\sigma}$ & $c_{\alpha}$ & $A \cup \operatorname{ic}(A) \cup \operatorname{ci}(A)$ & $c_{\sigma}$ \\
\hline$c_{\alpha}$ & $c_{\alpha}$ & $c_{\alpha}$ & $c_{\alpha}$ & $c_{\alpha}$ \\
\hline$c_{\pi}$ & $c_{\alpha}$ & $c_{\alpha}$ & $c_{\pi}$ & $c_{\pi}$ \\
\hline$c_{\beta}$ & $c_{\sigma}$ & $c_{\alpha}$ & $c_{\pi}$ & $c_{\beta}$ \\
\hline
\end{tabular}

Table 3

\begin{tabular}{|c||c|c|c|c|}
\hline$\circ$ & $c_{\sigma}$ & $c_{\alpha}$ & $c_{\pi}$ & $c_{\beta}$ \\
\hline \hline$i_{\sigma}$ & {$[A \cap \operatorname{cic}(A)] \cup \operatorname{ic}(A)$} & cic & ci & {$[A \cap \operatorname{ci}(A)] \cup \operatorname{ici}(A)$} \\
\hline$i_{\alpha}$ & ic & ic & ici & ici \\
\hline$i_{\pi}$ & ic & ic & {$[A \cup \operatorname{ci}(A)] \cap \operatorname{ic}(A)$} & {$[A \cap \operatorname{ic}(A)] \cup \operatorname{ici}(A)$} \\
\hline$i_{\beta}$ & $i_{\sigma} c_{\sigma}$ & cic & {$[A \cap \operatorname{cic}(A)] \cup \operatorname{ci}(A)$} & {$[A \cap \operatorname{cic}(A)] \cup \operatorname{ici}(A)$} \\
\hline
\end{tabular}

Table 4

\begin{tabular}{|c||c|c|c|c|}
\hline$\circ$ & $i_{\sigma}$ & $i_{\alpha}$ & $i_{\pi}$ & $i_{\beta}$ \\
\hline \hline$c_{\sigma}$ & $i_{\sigma} c_{\beta}$ & ici & ic & $i_{\sigma} c_{\sigma}$ \\
\hline$c_{\alpha}$ & $\mathrm{ci}$ & $\mathrm{ci}$ & $\mathrm{cic}$ & $\mathrm{cic}$ \\
\hline$c_{\pi}$ & $\mathrm{ci}$ & $\mathrm{ci}$ & {$[A \cap \mathrm{ic}(A)] \cup \operatorname{ci}(A)$} & $i_{\beta} c_{\pi}$ \\
\hline$c_{\beta}$ & $i_{\sigma} c_{\beta}$ & $\mathrm{ici}$ & $i_{\pi} c_{\beta}$ & $i_{\beta} c_{\beta}$ \\
\hline
\end{tabular}

follows. For our convenience we avoid writing $A$, that is, $i_{\sigma}(A)$ is written as simply $i_{\sigma}$ and so on.

$$
\begin{aligned}
& 1:=\mathrm{ici}, \quad 2:=\mathrm{ci}, \quad 3:=\mathrm{ic}, \\
& 4:=\mathrm{cic}, \quad 5:=i_{\alpha}, \quad 6:=i_{\sigma}, \\
& 7:=i_{\pi}, \quad 8:=i_{\beta}, \quad 9:=c_{\alpha}, \\
& 10:=c_{\pi}, \quad 11:=c_{\sigma}, \quad 12:=c_{\beta} \text {, }
\end{aligned}
$$

$$
13:=i_{\sigma} i_{\pi}=A \cap \operatorname{ic}(A) \cap \operatorname{ci}(A), \quad 14:=i_{\sigma} c_{\sigma}=[A \cap \operatorname{cic}(A)] \cup \operatorname{ic}(A),
$$




$$
\begin{array}{ll}
15:=i_{\sigma} c_{\beta}=[A \cap \operatorname{ci}(A)] \cup \operatorname{ici}(A), & 16:=i_{\pi} c_{\pi}=[A \cup \operatorname{ci}(A)] \cap \operatorname{ic}(A), \\
17:=i_{\pi} c_{\beta}=[A \cap \operatorname{ic}(A)] \cup \operatorname{ici}(A), & 18:=i_{\beta} c_{\pi}=[A \cap \operatorname{cic}(A)] \cup \operatorname{ci}(A), \\
19:=i_{\beta} c_{\beta}=[A \cap \operatorname{cic}(A)] \cup \operatorname{ici}(A), & 20:=c_{\sigma} c_{\pi}=A \cup \operatorname{ic}(A) \cup \operatorname{ci}(A), \\
21:=c_{\pi} i_{\pi}=[A \cap \operatorname{ic}(A)] \cup \operatorname{ci}(A) . &
\end{array}
$$

Now the question is: How many more new sets can we further obtain? Our investigation shows that at most 4 more new sets can be obtained by composing the above operators further. They are

$$
\begin{aligned}
& 22:=i_{\sigma} i_{\pi} c_{\pi}=\operatorname{ci}(A) \cap \operatorname{ic}(A), \\
& 23:=c_{\sigma} c_{\pi} i_{\pi}=\operatorname{ci}(A) \cup \operatorname{ic}(A), \\
& 24:=i_{\sigma} c_{\sigma} c_{\pi}=[A \cap \operatorname{cic}(A)] \cup \operatorname{ci}(A) \cup \operatorname{ic}(A), \\
& 25:=i_{\sigma} i_{\pi} c_{\beta}=[A \cap \operatorname{ci}(A) \cap \operatorname{ic}(A)] \cup \operatorname{ici}(A) .
\end{aligned}
$$

In the final exhaustive table, we show that these 25 sets are all the possible sets that can be obtained by using the operators $i_{\alpha}, i_{\sigma}, i_{\pi}, i_{\beta}, c_{\alpha}, c_{\sigma}, c_{\pi}$ and $c_{\beta}$. For our convenience, we use only the numerals to represent a set in the table. For

\begin{tabular}{|c|c|c|c|c|c|c|c|c|c|c|c|c|c|c|c|c|c|c|c|c|c|c|c|c|c|}
\hline 0 & 1 & 2 & 3 & 4 & 5 & 6 & 7 & 8 & 99 & 10 & 11 & 12 & 13 & 14 & 15 & 16 & 17 & 18 & 19 & 20 & 21 & 22 & 23 & 24 & 25 \\
\hline$\overline{11}$ & 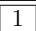 & $\overline{\overline{c 1}}$ & $\overline{3}$ & 3 & $\overline{11}$ & $\overline{\overline{11}}$ & $\overline{1}$ & $\overline{\overline{11}}$ & $\overline{c 3}$ & $\overline{11}$ & 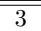 & $\bar{~} \overline{11}$ & $\overline{\overline{11}}$ & 3 & $\overline{\overline{11}}$ & $\overline{\overline{11}}$ & $\overline{\overline{11}}$ & $\overline{\overline{11}}$ & 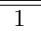 & 3 & 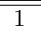 & $\bar{~} 1$ & $\overline{33}$ & 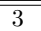 & 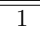 \\
\hline 2 & 2 & 2 & 4 & 4 & 2 & 2 & 2 & 2 & 4 & 2 & 4 & 2 & 2 & 4 & 2 & 2 & 2 & 2 & 2 & 4 & 2 & 2 & 4 & 4 & 2 \\
\hline 3 & 1 & 1 & 3 & 3 & 1 & 1 & 3 & 3 & 3 & 3 & 3 & 3 & 1 & 3 & 1 & 3 & 3 & 3 & 3 & 3 & 3 & 1 & 3 & 3 & 1 \\
\hline 4 & 2 & 2 & 4 & 4 & 2 & 2 & 4 & 4 & 4 & 4 & 4 & 4 & 2 & 4 & 2 & 4 & 4 & 4 & 4 & 4 & 4 & 2 & 4 & 4 & 2 \\
\hline 5 & 1 & 1 & 3 & 3 & 5 & 5 & 5 & 5 & 3 & 1 & 3 & 1 & 5 & 3 & 1 & 1 & 1 & 1 & 1 & 3 & 1 & 1 & 3 & 3 & 1 \\
\hline 6 & 1 & 2 & 3 & 4 & 5 & 6 & 13 & 6 & 4 & 2 & 14 & 15 & 13 & 14 & 15 & 22 & 25 & 2 & 15 & 24 & 2 & 22 & 23 & 24 & 25 \\
\hline 7 & 1 & 1 & 3 & 3 & 5 & 5 & 7 & 7 & 3 & 16 & 3 & 17 & 5 & 3 & 1 & 16 & 17 & 16 & 17 & 3 & 16 & 1 & 3 & 3 & 1 \\
\hline 8 & 1 & 2 & 3 & 4 & 5 & 6 & 7 & 8 & 4 & 18 & 14 & 19 & 13 & 14 & 15 & 16 & 17 & 18 & 19 & 24 & 21 & 22 & 23 & 24 & 25 \\
\hline 9 & 2 & 2 & 4 & 4 & 2 & 2 & 4 & 4 & 9 & 9 & 9 & 9 & 2 & 4 & 2 & 4 & 4 & 4 & 4 & 9 & 4 & 2 & 4 & 4 & 2 \\
\hline 10 & 2 & 2 & 4 & 4 & 2 & 2 & 21 & 18 & 9 & 10 & 9 & 10 & 2 & 4 & 2 & 21 & 21 & 18 & 18 & 9 & 21 & 2 & 4 & 4 & 2 \\
\hline 11 & 1 & 2 & 3 & 4 & 1 & 15 & 3 & 14 & 9 & 20 & 11 & 11 & 25 & 14 & 15 & 3 & 3 & 24 & 14 & 20 & 23 & 22 & 23 & 24 & 25 \\
\hline 12 & 1 & 2 & 3 & 4 & 1 & 15 & 17 & 19 & 9 & 10 & 11 & 12 & 25 & 14 & 15 & 16 & 17 & 18 & 19 & 20 & 21 & 22 & 23 & 24 & 25 \\
\hline 13 & 1 & 1 & 3 & 3 & 5 & 5 & 13 & 13 & 3 & 22 & 3 & 25 & 5 & 3 & 1 & 22 & 25 & 22 & 25 & 3 & 22 & 1 & 3 & 3 & 1 \\
\hline 14 & 1 & 2 & 3 & 4 & 1 & 15 & 3 & 14 & 4 & 24 & 14 & 14 & 25 & 14 & 15 & 3 & 3 & 24 & 14 & 24 & 23 & 22 & 23 & 24 & 25 \\
\hline 15 & 1 & 2 & 3 & 4 & 1 & 15 & 25 & 15 & 4 & 2 & 14 & 15 & 25 & 14 & 15 & 22 & 25 & 2 & 15 & 24 & 2 & 22 & 23 & 24 & 25 \\
\hline 16 & 1 & 1 & 3 & 3 & 1 & 1 & 16 & 16 & 3 & 16 & 3 & 16 & 1 & 3 & 1 & 16 & 16 & 16 & 16 & 3 & 16 & 1 & 3 & 3 & 1 \\
\hline 17 & 1 & 1 & 3 & 3 & 1 & 1 & 17 & 17 & 3 & 16 & 3 & 17 & 1 & 3 & 1 & 16 & 17 & 16 & 17 & 3 & 16 & 1 & 3 & 3 & 1 \\
\hline 18 & 2 & 2 & 4 & 4 & 2 & 2 & 21 & 18 & 4 & 18 & 4 & 18 & 2 & 4 & 2 & 21 & 21 & 18 & 18 & 4 & 21 & 2 & 4 & 4 & 2 \\
\hline 19 & 1 & 2 & 3 & 4 & 1 & 15 & 17 & 19 & 4 & 18 & 14 & 19 & 25 & 14 & 15 & 16 & 17 & 18 & 19 & 24 & 21 & 22 & 23 & 24 & 25 \\
\hline 20 & 2 & 2 & 4 & 4 & 2 & 2 & 23 & 24 & 9 & 20 & 9 & 20 & 2 & 4 & 2 & 23 & 23 & 24 & 24 & 9 & 23 & 2 & 4 & 4 & 2 \\
\hline 21 & 2 & 2 & 4 & 4 & 2 & 2 & 21 & 21 & 4 & 21 & 4 & 21 & 2 & 4 & 2 & 21 & 21 & 21 & 21 & 4 & 21 & 2 & 4 & 4 & 2 \\
\hline 22 & 1 & 1 & 3 & 3 & 1 & 1 & 22 & 22 & 3 & 22 & 3 & 22 & 1 & 3 & 1 & 22 & 22 & 22 & 22 & 3 & 22 & 1 & 3 & 3 & 1 \\
\hline 23 & 2 & 2 & 4 & 4 & 2 & 2 & 23 & 23 & 4 & 23 & 4 & 23 & 2 & 4 & 2 & 23 & 23 & 23 & 23 & 4 & 23 & 2 & 4 & 4 & 2 \\
\hline 24 & 2 & 2 & 4 & 4 & 2 & 2 & 23 & 24 & 4 & 24 & 4 & 24 & 2 & 4 & 2 & 23 & 23 & 24 & 24 & 4 & 23 & 2 & 4 & 4 & 2 \\
\hline 25 & 1 & 1 & 3 & 3 & 1 & 1 & 25 & 25 & 3 & 22 & 3 & 25 & 1 & 3 & 1 & 22 & 25 & 22 & 25 & 3 & 22 & 1 & 3 & 3 & 1 \\
\hline
\end{tabular}
example, 1,2 and 3 represent $\operatorname{ici}(A), \operatorname{ci}(A)$ and ic $(A)$, respectively as they have been listed above. Verification of the calculations involved in preparing the composition table is not much difficult (it is based on Lemma 2.3, the above tables and the Kuratowski's closure-complement theorem) and is left to the reader. The authors have also verified the associativity of the compositions using "Light's associativity test" [3].

Table 5 
Below we provide an example of a topological space, where the bound of 25 sets has been demonstrated.

Example 2.5. Let $X=\mathbb{R}$ be the set of real numbers with the usual topology. Let $A \subseteq X$ be defined by

$$
\begin{aligned}
A=\{-1 / n, n \in \mathbb{N}\} & \cup[[1,3] \backslash\{2+1 / n, n \in \mathbb{N}\}] \\
\cup & {\left[(5,7] \cap\left(\mathbb{Q} \cup \bigcup_{n=1}^{\infty}\left(6+\frac{1}{2 n \pi}, 6+\frac{1}{(2 n-1) \pi}\right)\right] \cup(-3,-2] .\right.}
\end{aligned}
$$

Then, we have

(i)

$$
\operatorname{ic}(A)=(1,3) \cup(5,7) \cup(-3,-2) ;
$$

(ii)

$$
\operatorname{cic}(A)=[1,3] \cup[5,7] \cup[-3,-2] ;
$$

(iii)

$$
\operatorname{ci}(A)=[1,3] \cup\{6\} \cup \bigcup_{n=1}^{\infty}\left[6+\frac{1}{2 n \pi}, 6+\frac{1}{(2 n-1) \pi}\right] \cup[-3,-2]
$$

(iv)

$$
\operatorname{ici}(A)=(1,3) \cup \bigcup_{n=1}^{\infty}\left(6+\frac{1}{2 n \pi}, 6+\frac{1}{(2 n-1) \pi}\right) \cup(-3,-2) ;
$$

( v)

$$
\begin{aligned}
A \cap \operatorname{ic}(A) & =(1,3) \backslash\{2+1 / n, n \in \mathbb{N}\} \\
& \cup\left[(5,7) \cap\left(\mathbb{Q} \cup \bigcup_{n=1}^{\infty}\left(6+\frac{1}{2 n \pi}, 6+\frac{1}{(2 n-1) \pi}\right)\right)\right] \cup(-3,-2) ;
\end{aligned}
$$

(vi)

$$
A \cup \operatorname{ic}(A)=\{-1 / n, n \in \mathbb{N}\} \cup[1,3) \cup(5,7] \cup(-3,-2] ;
$$

(vii)

$$
\begin{aligned}
A \cap \operatorname{cic}(A) & =[1,3] \backslash\{2+1 / n, n \in \mathbb{N}\} \\
& \cup\left[(5,7] \cap\left(\mathbb{Q} \cup \bigcup_{n=1}^{\infty}\left(6+\frac{1}{2 n \pi}, 6+\frac{1}{(2 n-1) \pi}\right)\right] \cup(-3,-2] ;\right.
\end{aligned}
$$

(viii)

$$
A \cup \operatorname{cic}(A)=\{-1 / n, n \in \mathbb{N}\} \cup[1,3] \cup[5,7] \cup[-3,-2] ;
$$

(ix)

$$
\begin{aligned}
A \cap \operatorname{ci}(A) & =[1,3] \backslash\{2+1 / n, n \in \mathbb{N}\} \cup\{6\} \\
& \cup \bigcup_{n=1}^{\infty}\left(6+\frac{1}{2 n \pi}, 6+\frac{1}{(2 n-1) \pi}\right] \cup(-3,-2]
\end{aligned}
$$


(x)

$$
\begin{aligned}
A \cup \operatorname{ci}(A) & =\{-1 / n, n \in \mathbb{N}\} \cup[1,3] \\
& \cup\left[(5,7] \cap\left(\mathbb{Q} \cup \bigcup_{n=1}^{\infty}\left[6+\frac{1}{2 n \pi}, 6+\frac{1}{(2 n-1) \pi}\right]\right)\right] \cup[-3,-2] ;
\end{aligned}
$$

(xi)

$A \cap \operatorname{ici}(A)=(1,3) \backslash\{2+1 / n, n \in \mathbb{N}\} \cup \bigcup_{n=1}^{\infty}\left(6+\frac{1}{2 n \pi}, 6+\frac{1}{(2 n-1) \pi}\right) \cup(-3,-2)$; (xii)

$A \cup \operatorname{ici}(A)=\{-1 / n, n \in \mathbb{N}\} \cup[1,3)$

$$
\cup\left[(5,7] \cap\left(\mathbb{Q} \cup \bigcup_{n=1}^{\infty}\left(6+\frac{1}{2 n \pi}, 6+\frac{1}{(2 n-1) \pi}\right]\right)\right] \cup(-3,-2] ;
$$

(xiii)

$$
\operatorname{ic}(A) \cap \operatorname{ci}(A)=(1,3) \cup\{6\} \cup \bigcup_{n=1}^{\infty}\left[6+\frac{1}{2 n \pi}, 6+\frac{1}{(2 n-1) \pi}\right] \cup(-3,-2) ;
$$

(xiv)

(xv)

$$
\operatorname{ic}(A) \cup \operatorname{ci}(A)=[1,3] \cup(5,7) \cup[-3,-2] ;
$$

$$
\begin{aligned}
A \cap \operatorname{ic}(A) \cap \operatorname{ci}(A) & =(1,3) \backslash\{2+1 / n, n \in \mathbb{N}\} \cup\{6\} \\
& \cup \bigcup_{n=1}^{\infty}\left(6+\frac{1}{2 n \pi}, 6+\frac{1}{(2 n-1) \pi}\right] \cup(-3,-2) ;
\end{aligned}
$$

(xvi)

(xvii)

$$
A \cup \operatorname{ic}(A) \cup \operatorname{ci}(A)=\{-1 / n, n \in \mathbb{N}\} \cup[1,3] \cup(5,7] \cup[-3,-2] ;
$$

(xviii)

$$
[A \cap \operatorname{cic}(A)] \cup \operatorname{ic}(A)=[1,3) \cup(5,7] \cup(-3,-2] ;
$$

$$
[A \cap \operatorname{ci}(A)] \cup \operatorname{ici}(A)=[1,3) \cup\{6\} \cup \bigcup_{n=1}^{\infty}\left(6+\frac{1}{2 n \pi}, 6+\frac{1}{(2 n-1) \pi}\right] \cup(-3,-2] ;
$$
(xix)

$[A \cup \operatorname{ci}(A)] \cap \operatorname{ic}(A)=(1,3)$

$$
\cup\left[(5,7) \cap\left(\mathbb{Q} \cup \bigcup_{n=1}^{\infty}\left[6+\frac{1}{2 n \pi}, 6+\frac{1}{(2 n-1) \pi}\right]\right)\right] \cup(-3,-2) ;
$$

$(\mathrm{xx})$

$[A \cap \operatorname{ic}(A)] \cup \operatorname{ici}(A)=(1,3)$

$$
\cup\left[(5,7) \cap\left(\mathbb{Q} \cup \bigcup_{n=1}^{\infty}\left(6+\frac{1}{2 n \pi}, 6+\frac{1}{(2 n-1) \pi}\right)\right)\right] \cup(-3,-2) ;
$$


(xxi)

$[A \cap \operatorname{cic}(A)] \cup \operatorname{ci}(A)=[1,3]$

$$
\cup\left[(5,7] \cap\left(\mathbb{Q} \cup \bigcup_{n=1}^{\infty}\left[6+\frac{1}{2 n \pi}, 6+\frac{1}{(2 n-1) \pi}\right]\right)\right] \cup[-3,-2] ;
$$

(xxii)

$[A \cap \operatorname{cic}(A)] \cup \operatorname{ici}(A)=[1,3)$

$$
\cup\left[(5,7] \cap\left(\mathbb{Q} \cup \bigcup_{n=1}^{\infty}\left(6+\frac{1}{2 n \pi}, 6+\frac{1}{(2 n-1) \pi}\right]\right)\right] \cup(-3,-2] ;
$$

(xxiii)

$$
\begin{aligned}
{[A \cap \operatorname{ic}(A)] \cup \operatorname{ci}(A) } & =[1,3] \\
& \cup\left[(5,7) \cap\left(\mathbb{Q} \cup \bigcup_{n=1}^{\infty}\left[6+\frac{1}{2 n \pi}, 6+\frac{1}{(2 n-1) \pi}\right]\right)\right] \cup[-3,-2] ;
\end{aligned}
$$

(xxiv)

$$
[A \cup \operatorname{ci}(A) \cup \operatorname{ic}(A)] \cap \operatorname{cic}(A)=[1,3] \cup(5,7] \cup[-3,-2] ;
$$

$(\mathrm{xxv})$

$$
\begin{aligned}
{[A \cap \operatorname{ci}(A) \cap \operatorname{ic}(A)] \cup \operatorname{ici}(A) } & =(1,3) \cup\{6\} \\
& \cup \bigcup_{n=1}^{\infty}\left(6+\frac{1}{2 n \pi}, 6+\frac{1}{(2 n-1) \pi}\right] \cup(-3,-2) .
\end{aligned}
$$

\section{Some SPeCial CASES}

If the topology on $X$ satisfies some extra conditions, then the upper bound obtained above may be less than 25. We have the following results.

Definition 3.1. A topological space $(X, \tau)$ is said to be

(i) extremally disconnected [16] if the closure of any open set is open,

(ii) resolvable [10] if it contains a dense set with empty interior,

(iii) open unresolvable [2] if no open subspace is resolvable,

(iv) partition space [18] if its open sets form a Boolean algebra.

For open unresolvable space, we use the notation $O U$-space.

Corollary 3.2. In the case of $(X, \tau)$ being extremally disconnected (OU-space, partition space, extremally disconnected $O U$-space or discrete space), the upper bound obtained above reduces to 7 (resp. 7, 3, 3 or 1).

Case 1. Let $(X, \tau)$ be extremally disconnected and $A \subseteq X$. Then $\operatorname{ci}(A)$ is an open set. Hence, $\operatorname{ici}(A)=\operatorname{ci}(A)$ and $\operatorname{cic}(A)=\operatorname{ic}(A)$. Thus the distinct sets obtained from the operators $i_{\gamma}, c_{\gamma}($ where $\gamma=\alpha, \pi, \sigma, \beta)$ are ici $=\mathrm{ci}$, ic $=\mathrm{cic}, i_{\alpha}=i_{\sigma}, i_{\pi}=$ $i_{\beta}, c_{\sigma}=c_{\alpha}, c_{\pi}=c_{\beta}$ and $i_{\pi} c_{\pi}=[A \cup \operatorname{ci}(A)] \cap \mathrm{ic}(A)$, which are seven in number altogether. 
Case 2. Let $(X, \tau)$ be an $O U$-space and $A \subseteq X$. In [2], Aull shows that $O U$-spaces are exactly those spaces in which every dense set has dense interior and we know that spaces whose dense sets have dense interiors satisfy ici $=$ ic. For the converse part, let ici $=$ ic for every $A \subseteq X$ and let $A$ be a dense set in $X$. Therefore, $c(A)=X$ and $\operatorname{ic}(A)=X$. Since $\operatorname{ici}(A)=\operatorname{ic}(A)=X$, we have $X \subseteq \operatorname{ci}(A)$. Hence, $i(A)$ is dense in $X$. Therefore, in an $O U$-space, we have ici $=$ ic and cic $=$ ci. Thus the distinct sets obtained from the operators $i_{\gamma}, c_{\gamma}$ (where $\left.\gamma=\alpha, \pi, \sigma, \beta\right)$ are $\mathrm{ici}=\mathrm{ic}, \mathrm{ci}=\mathrm{cic}, i_{\alpha}=i_{\pi}, i_{\sigma}=i_{\beta}, c_{\sigma}=c_{\beta}, c_{\pi}=c_{\alpha}$ and $i_{\sigma} c_{\beta}=[A \cap \operatorname{ci}(A)] \cup \operatorname{ic}(A)$, which are seven in number altogether.

Case 3. Let $(X, \tau)$ be a partition-space. Then its open sets are clopen, that is, closed and open simultaneously. Therefore, $\operatorname{ci}(A)=\mathrm{i}(A)$ for $A \subseteq X$. Therefore, in partition-space, we have $\mathrm{i}=\mathrm{ici}=\mathrm{ci}$ and $\mathrm{cic}=\mathrm{ic}=\mathrm{c}$. Thus the distinct sets obtained from the operators $i_{\gamma}, c_{\gamma}($ where $\gamma=\alpha, \pi, \sigma, \beta)$ are i, c and $A$ only.

Case 4. Let $(X, \tau)$ be an extremally disconnected $O U$-space. Then we have ici $=$ $\mathrm{ci}=\mathrm{ic}=\mathrm{cic}$ for $A \subseteq X$. Therefore, in extremally disconnected $O U$-space, the distinct sets obtained from the operators $i_{\gamma}, c_{\gamma}($ where $\gamma=\alpha, \pi, \sigma, \beta)$ are ici, $i_{\alpha}$ and $c_{\alpha}$ only, which are three in number altogether.

Case 5. Let $(X, \tau)$ be a discrete space. Then every singleton is closed and open. Therefore, $\mathrm{c}(A)=\mathrm{i}(A)=A$ for $A \subseteq X$. Therefore, in discrete space, we have only 1 set $A$ itself, from the operators $i_{\gamma}, c_{\gamma}($ where $\gamma=\alpha, \pi, \sigma, \beta)$.

Acknowledgement. The authors thank the referee for his/her valuable suggestions which helped us improve the quality of our paper.

\section{REFERENCES}

[1] M. E. Abd EI-Monsef, R. A. Mahmoud and S. N. El-Deeb, $\beta$-open sets and $\beta$-continuous mappings, Bull. Fac. Sci. Assiut Univ. 12 (1966), 77-90.

[2] C. E. Aull, Classification of topological spaces, Bull. de l'Acad. Pol. Sci. Math. Astron. Phys. 15 (1967), 773-778.

[3] A. R. Bednarek, An extension of Light's associativity test, Amer. Math. Monthly 75 (1968), 531-532.

[4] J. Brzozowski, E. Grant and J. Shallit, Closures in Formal Languages and Kuratowski's Theorem, Developments in Language Theory, Lecture Notes in Computer Science 5583, Springer, 2009.

[5] A. V. Chagrov, Kuratowski numbers (Russian), Application of functional analysis in approximation theory, Kalinin. Gos. Univ., Kalinin, 1982, 186-190.

[6] Á. Császár, Generalized open sets, Acta Math. Hungar. 75 (1997), 65-87.

[7] Á. Császár, On the $\gamma-$ interior and $\gamma-$ closure of a set, Acta Math. Hungar. 80 (1998), 89-93.

[8] Á. Császár, Generalized topology, generalized continuity, Acta Math. Hungar. 96 (2002), 351-357.

[9] B. J. Gardner and M. Jackson, The Kuratowski closure complement theorem, New Zealand J. Math. 38 (2008), 9-44.

[10] E. Hewitt, A problem in set-theoretic topology, Duke Math. J. 10 (1943), 309-333.

[11] C. Kuratowski, Sur l'operation A de l'analysis situs, Fund. Math. 3 (1922), 182-199.

[12] N. Levine, Semi-open sets and semi-continuity in topological spaces, Amer. Math. Monthly 70 (1963), 36-41.

[13] A. S. Mashhour, M. E. Abd EI-Monsef and S. N. El-Deeb, On precontinuous and weak precontinuous mappings, Proc. Math. and Phys. Soc. Egypt 53 (1982) 47-53. 
[14] O. Njåstad, On some classes of nearly open sets, Pac. J. Math. 15 (1965), 961-970.

[15] D. Peleg, A Generalized closure and complement phenomenon, Discrete Math. 50 (1984), 285-293.

[16] R. D. Sarma, On extremally disconnected generalized topologies, Acta Math. Hungar. 134 (2012), 583-588.

[17] R. D. Sarma and Ankit Gupta, On the k-numbers of some generalized Kuratowski operators in topology, in: The proceedings of the $3^{\text {rd }}$ International Conference on "Innovative Approach in Applied Physical, Mathematical/statistical, Chemical Science" and Emerging Energy Technology for Sustainable Development, 2014, 50-54.

[18] L. A. Steen and J. A. Seebach Jr., Counterexamples in Topology, Holt, Rinehart and Winston Inc., New-York, 1970.

Ankit Gupta, Department of Mathematics, University of Delhi, Delhi 110007, India e-mail: ankitsince1988@yahoo.co.in

Ratna Dev Sarma, Department of Mathematics, Rajdhani College, University of Delhi, Delhi 110015, India

e-mail: ratna_sarma@yahoo.com 